\title{
La regionalización de la atención de cardiopatías congénitas: una meta pendiente
}

\author{
Regionalization of congenital heart disease care: a pending goal \\ Juan Calderón-Colmenero* \\ Departamento de Cardiología Pediátrica, Instituto Nacional de Cardiología Ignacio Chávez. Ciudad de México, México
}

\begin{abstract}
Resumen
Las cardiopatías congénitas son consideradas las malformaciones más frecuentes. En México cada año entre 12 y 16 mil niños nacen con algún tipo de cardiopatía congénita, y es la segunda causa de mortalidad en menores de 1 año y en niños de entre 1 y 4 años. Se analiza la problemática de la atención de las cardiopatías congénitas desde una perspectiva de políticas de salud en el país, con énfasis en lograr regionalizar su atención con el fin de racionalizar la utilización de los recursos disponibles y con el objetivo de atender al mayor número de pacientes y lograr los mejores resultados clínicos. Se enfatiza la necesidad de promover la cooperación entre las diferentes organizaciones de la sociedad civil y las instituciones para intercambiar ideas que permitan crear consensos y proyectos conjuntos para una mejor utilización de los recursos disponibles para lograr la atención universal y de calidad de los niños con cardiopatía congénita en todo el país.
\end{abstract}

Palabras clave: Cardiopatías congénitas. Cirugía cardíaca. Cateterismo intervencionista. Mortalidad infantil. Políticas de salud. México.

\begin{abstract}
Congenital heart diseases are considered the most frequent malformations. In Mexico every year between 12 and 16 thousand children are born with some type of congenital heart disease and it is the second cause of mortality in children under 1 year of age and in children aged between 1 and 4 years. The problem of the care of congenital heart diseases is analyzed from a perspective of health policies in the country with an emphasis on regionalizing their care in order to rationalize the use of available resources and with the aim of serving the largest number of patients and achieve the best clinical results. It emphasizes the need to promote cooperation among the different civil society organizations and institutions to exchange ideas to create consensus and joint projects for a better use of available resources to achieve universal and quality care of children with congenital heart disease in all the country.
\end{abstract}

Key words: Congenital heart disease. Heart surgery. Interventional catheterization. Child mortality. Health Policies. Mexico.

\section{Correspondencia:}

Juan Calderón-Colmenero

Juan Badiano, 1

Col. Sección XVI, Del. Tlalpan

Fecha de recepción: 24-09-2018

C.P. 14080, Ciudad de México, México

E-mail: juanecalderon @yahoo.com.mx
Disponible en internet: 08-05-2019 Arch Cardiol Mex. 2019;89(2):150-159 www.archivoscardiologia.com 1405-9940 @ 2019 Instituto Nacional de Cardiología Ignacio Chávez. Publicado por Permanyer México SA de CV. Este es un artículo Open Access bajo la licencia CC BY-NC-ND (http://creativecommons.org/licenses/by-nc-nd/4.0/). 
«La pobreza es una privación de ciertas capacidades, una de las cuales es la salud, entonces, por definición, todo lo que se haga por mejorar la salud reduce la pobreza.»

\section{Sir George Alleyne}

Si bien en México hay indudables avances en indicadores sociales: la esperanza de vida, pasó de alrededor de 36 años en 1910 a 75.3 años en 2017; la mortalidad en lactantes < 1 año disminuyó de 37 por 1,000 nacidos vivos en 1990 a 12 por 1,000 nacidos vivos en el 2016; el cumplimiento del programa de vacunación es del $98 \%$, superior a países como Italia, EE.UU. y Reino Unido. A pesar de los logros enumerados, nuestro país se encuentra en un punto crítico en el ámbito de la salud y requiere romper con inercias, realizar un profundo análisis de nuestros problemas y emprender acciones novedosas (Fig. 1 y 2).

En el año 2000 alrededor del $60 \%$ de la población mexicana contaba con acceso al Instituto Mexicano del Seguro Social (IMSS) o al Instituto de Seguridad y Servicios Sociales de los Trabajadores del Estado (ISSSTE); el Ejército, la Marina y Pemex (Petróleos Mexicanos) tenían servicios de salud propios; un pequeño porcentaje tenían algún seguro médico privado $(<3 \%)$ y el resto de la población carecía de algún tipo de seguro para servicios de salud. Knaul, et al. ${ }^{1}$ estimaron que el $63 \%$ de las familias, la mayoría pobres y sin seguridad social, había tenido que erogar gastos muy significativos, lo que había condicionado un mayor empobrecimiento. También fue estimado que el $58 \%$ del gasto total en salud provenía de los hogares por medio del gasto de bolsillo. México es el segundo país miembro de la Organización para la Cooperación y el Desarrollo Económico (OCDE), detrás de Letonia, con el mayor porcentaje de gasto en bolsillo en salud, definido como el gasto directo de las familias para solventar requerimientos de la atención de la salud, sin incluir seguros médicos privados ${ }^{2}$. De acuerdo con información de la Organización Mundial de la Salud, la inversión en salud en nuestro país era del $4.9 \%$ del producto interno bruto (PIB) en el año 2000, se elevó al $6.2 \%$ en el 2009 y disminuyó al $5.9 \%$ en el $2015^{3}$. La inversión en salud promedio o gasto de salud de los países de Latinoamérica es del 7\% y de los países de la OCDE es del $8.9 \%{ }^{4}$. Por otra parte, Cano Valle, et al. ${ }^{4}$ refieren que de acuerdo con la OCDE (2012), el gasto administrativo en México, en el que se incluye acciones de rectoría y gestión en el Sistema Nacional de Salud, se ubica cercano al $10 \%$ del gasto total en salud pública, 2.5 veces más que el promedio de los países de la OCDE, que es del $4 \%$. Lo anterior nos muestra una inversión en salud insuficiente, aunada a un gasto administrativo elevado e ineficiente ${ }^{5,6}$. Esta situación no es privativa de nuestro país, David Cutler, profesor de Economía Aplicada en Harvard College, muy reconocido en el campo de la salud, señaló en un ensayo que los recursos invertidos en servicios de salud en EE.UU. que no generan mejora en los servicios en salud ascienden al $30 \%$ del gasto total; según Julio Frenk, et al. dicho monto equivale al valor total de la economía de México ${ }^{7,8}$.

Se ha mencionado que por cada $10 \%$ de aumento en la esperanza de vida se incrementa el PIB en un $4 \%{ }^{9}$. Es primordial establecer una relación entre desarrollo económico y salud, modificar paradigmas para hacer que el núcleo de atención sea una población saludable que pueda dinamizar la economía y como consecuencia, en un círculo armonioso, provoque que se invierta en mayor bienestar ${ }^{10}$. En nuestro país para acrecentar la población con algún tipo de seguro médico y disminuir el gasto de bolsillo de las familias se creó el Seguro Popular en el 2003, que en la actualidad tiene afiliadas a 53 millones de personas. Esta entidad funciona como un órgano autónomo de la Secretaría de Salud y cuenta con un paquete de servicios conocido como Catálogo Universal de Servicios de Salud (CAUSES), el cual contempla, en la actualidad, 287 intervenciones además del Fondo de Gastos Catastróficos, que incluye 149 intervenciones de alto costo. De acuerdo con cifras de la Secretaría de Salud y de la Encuesta Intercensal 2015, el número de mexicanos con seguro público de salud pasó de cerca de 40 millones ( $41 \%$ de la población total) a 98 millones en 2015 (82\% de la población total) ${ }^{10-12}$. En el caso de las malformaciones congénitas del corazón, en el fondo de gastos catastróficos está contemplada su atención quirúrgica o mediante cateterismo intervencionista y si bien ha tenido diferentes reglamentaciones, en los últimos tres sexenios ha posibilitado que se ayude a estos pacientes y que no represente un gasto de bolsillo importante para las familias y una devastación financiera a las instituciones de salud. Con sustento en cálculos actuariales, el Seguro Popular realiza un pago a la institución prestadora del servicio por cada paciente con cardiopatía congénita (CC) atendido a quien se le realizó un procedimiento quirúrgico o intervencionista de $\$ 100,743.00$. Sería conveniente que el monto se ajustara en relación con la complejidad de los pacientes atendidos con base en los métodos de estratificación de riesgo existentes: RACHS-1 (Risk Adjustment in Congenital Heart Surgery), Aristóteles, STS-EACTS (Society of Thoracic Surgeons-European Association 


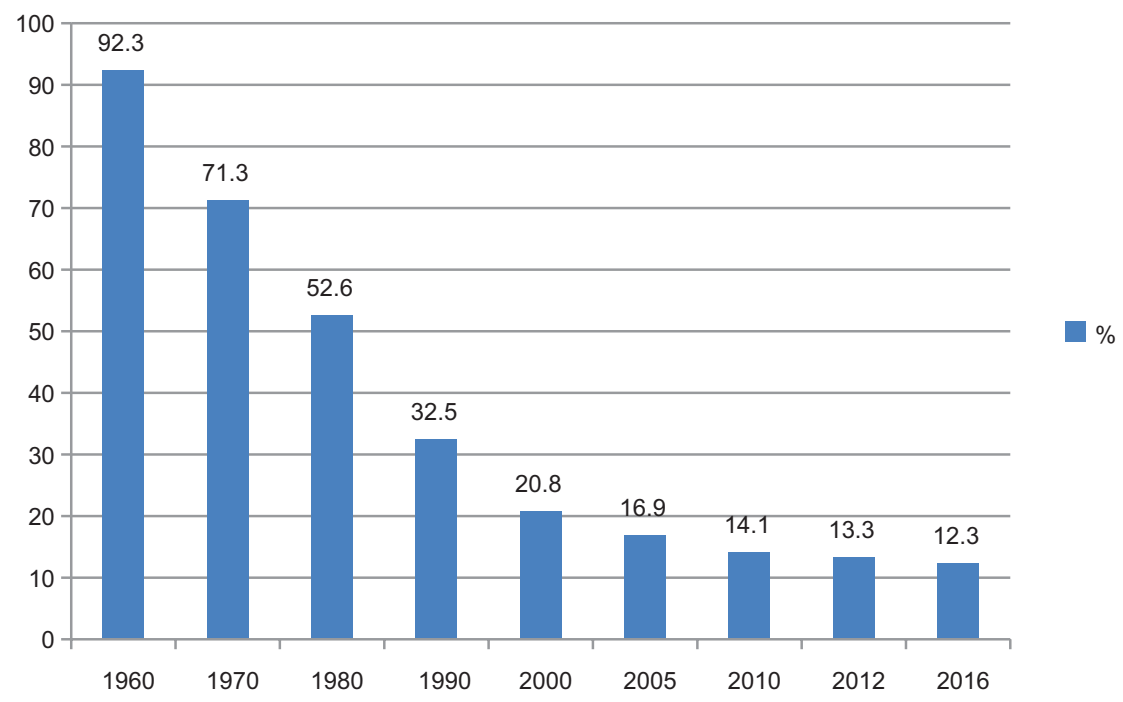

Figura 1. Mortalidad Infantil en menores de 1 año ladaptada dela Organization for Economic Co-operation and Development [OECD]. MEXICO Country Highlights [OECD], Doing Better for Children ${ }^{13}$.

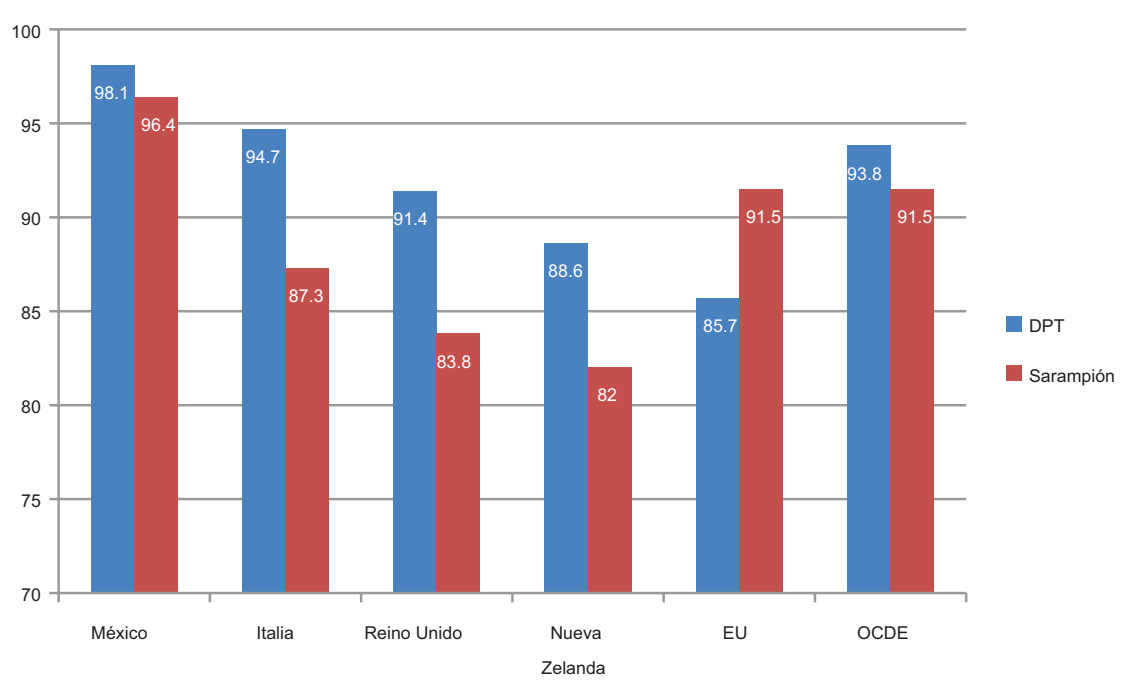

Figura 2. Porcentaje de cumplimiento en vacunación (adaptada de IndexMundi) ${ }^{14}$.

DPT: difteria, pertusis, tétanos; OCDE: Organización para la Cooperación y el Desarrollo Económicos.

for Cardiothoracic Surgery); esta corrección sería de suma importancia para aquellas instituciones que manejan un mayor volumen de pacientes con CC de mayor complejidad ${ }^{15,16}$.

En el año 2010 publicamos el artículo Problemática de las cardiopatías congénitas en México. Propuesta de Regionalización, en el cual se abordó por primera vez la problemática desde una perspectiva del país y se propuso regionalizar su atención con el fin de hacer mejor uso de los recursos disponibles, con el objetivo de atender al mayor número de pacientes y lograr los mejores resultados clínicos ${ }^{17}$. 


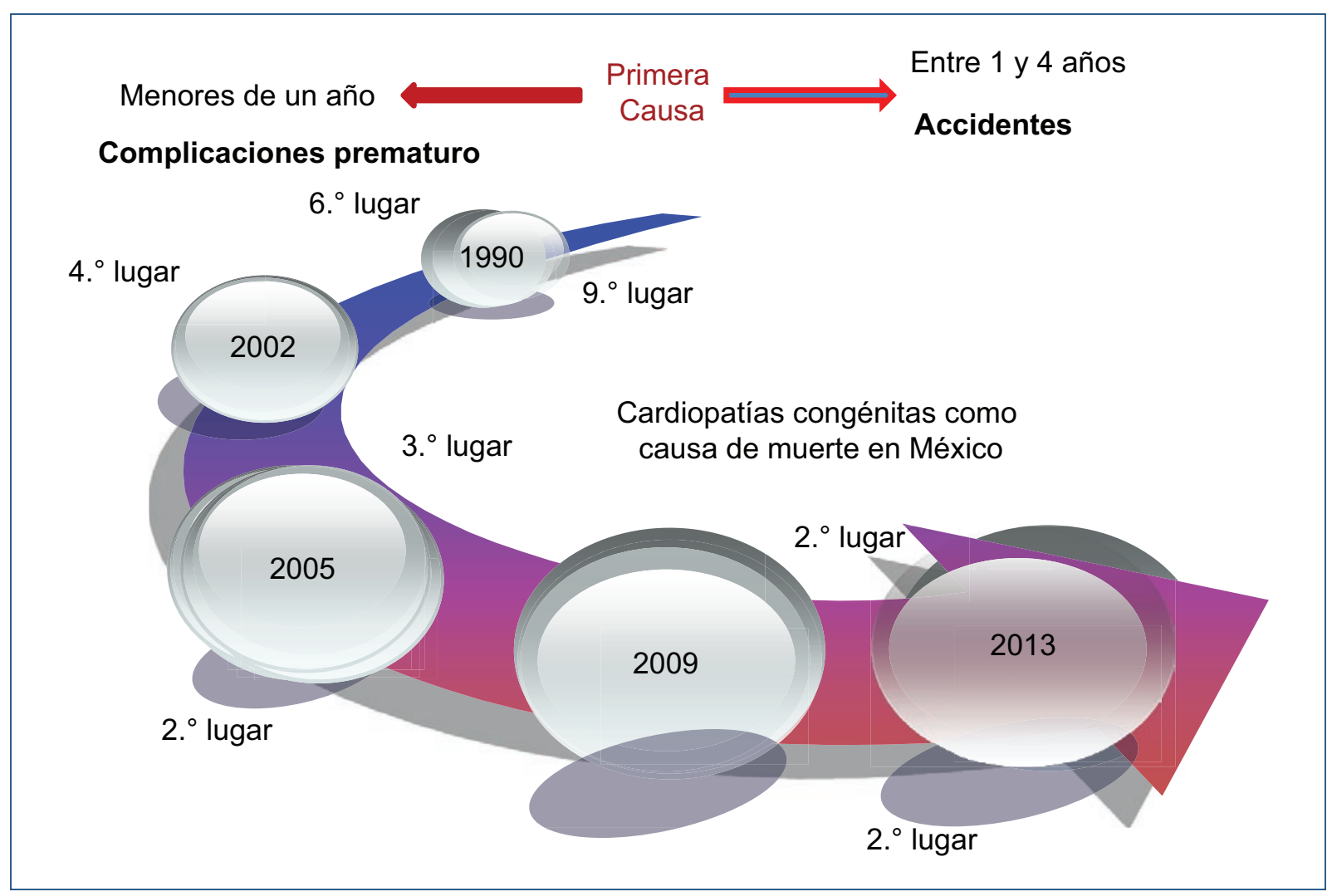

Figura 3. Causas de Mortalidad en México.

Las CC son consideradas las malformaciones más frecuentes y fueron definidas por Mitchell, et al..$^{18}$ como «anomalías estructurales evidentes del corazón o de los grandes vasos intratorácicos con una repercusión real o potencial». La prevalencia referida de CC por 1,000 recién nacidos vivos va de 2.17 en Canadá a 10.6 en Japón y 12.3 en Italia. Estas diferencias en la prevalencia se pueden explicar por el momento en que se realiza el muestreo, ya sea en recién nacidos 0 en menores de 1 año de vida; o a que sean incluidas patologías como la aorta bivalva presente entre el 1 al $2 \%$ de la población o comunicaciones interventriculares minúsculas con una incidencia del 2 al $5 \%$ y que tienen un cierre espontáneo hasta en el $90 \%$ de los niños, y los conductos arteriosos silentes sin repercusión hemodinámica ${ }^{19,20}$.

Si bien desconocemos la prevalencia de las CC en México, haciendo una extrapolación a partir de la prevalencia mundial reportada y conociendo el número de nacimientos anuales (alrededor de 2 millones), se puede afirmar que nacen entre 12 a 16 mil niños con CC; otra forma de establecer su trascendencia es por la tasa de mortalidad, en 1990 se ubicaba en el sexto lugar como causa de muerte en los menores de un año y a partir de 2005 como la segunda causa de mortalidad, donde permanece hasta la actualidad; en el grupo etario de 1 a 4 años es desde el 2009 la segunda causa de mortalidad (Fig. 3). En la investigación de la Dra. Teresa Murguía-Peniche, et al. ${ }^{21}$, se encontró que de 1988 a 2013 la mortalidad infantil secundaria a CC en toda la república se incrementó en $24.8 \%$, con un aumento en el número de decesos de 114.4 a 146.4 por 100 mil recién nacidos. En el último año del estudio (2013) se documentó un total de 3,593 decesos consecutivos a CC; un tercio de ellos ocurrió durante la primera semana de vida y los factores de riesgo encontrados fueron ser del sexo masculino, nacer en hospitales no institucionales y en zonas rurales. Los autores concluyeron que las $\mathrm{CC}$ son un serio problema de salud pública en nuestro país, que se requiere de una detección temprana y un monitoreo epidemiológico. Existe otro estudio, con un abordaje similar al anterior, en el cual el investigador Sánchez-Barriga ${ }^{22}$ analizó la mortalidad por malformaciones congénitas del corazón y grandes arterias en el periodo del 2000 al 2015 , encontrando que 70,741 individuos, entre niños 
y adultos, fallecieron por esa causa en el lapso mencionado, la tasa de mortalidad ajustada a la edad por cada 100,000 se elevó de 3.3 a 4; con respecto a los menores de 1 año, la tasa de mortalidad por 100,000 nacidos vivos se incrementó de 143.9 a 217.3. Es de mencionar que la región con mayor mortalidad fue la región socioeconómica 7 (Ciudad de México), según la clasificación del Instituto Nacional de Estadística y Geografía (INEGI). El investigador afirma que el incremento en la mortalidad se puede explicar por una mejoría en el diagnóstico de estas patologías; por mi parte, considero que la mayor mortalidad que se puede explicar en la Ciudad de México es reflejo de la gran concentración de los servicios médicos de alta especialidad.

Las recomendaciones del Comité de Cardiopatías Congénitas de la Asociación Europea de Cirujanos Cardiotorácicos (EACTS, por sus siglas en inglés) establecen la necesidad de un hospital con capacidad para llevar a cabo cirugía cardiovascular por cada cuatro millones de habitantes. En el 2010, México tenía una población de 103 millones de habitantes y requería de al menos de 25 centros de cirugía cardíaca para la adecuada atención de las CC en el país; en ese entonces existían 11 centros hospitalarios con actividad de cirugía cardíaca pediátrica consistente, 8 de los cuales ubicados en la Ciudad de México. En el 2015, con una población de 119 millones de habitantes de acuerdo con la información proporcionada por el INEGI, los centros con capacidad para llevar a cabo cirugías cardíacas pediátricas necesarias deberían ser por lo menos $30^{23}$.

En la propuesta de regionalización se consideró que la distribución de los centros para la atención medicoquirúrgica de CC debía sustentarse en las siguientes premisas: a) la tasa de mortalidad por malformaciones del corazón y grandes arterias en $<1$ año de cada uno de los Estados del país; b) aprovechar hospitales existentes con infraestructura que permita en el menor plazo fortalecerlos tecnológicamente y con recursos humanos; c) dar énfasis en regiones con una elevada tasa de natalidad, y d) priorizar zonas en que el traslado de los pacientes por factores tanto orográficos como socioeconómicos y culturales sea complicado.

Por su parte, la Secretaría de Salud, con el objetivo de ampliar la cobertura a nivel nacional de servicios especializados dirigidos a atender padecimientos de poca frecuencia y alta complejidad diagnóstica y terapéutica, decidió la edificación de Hospitales Regionales de Alta Especialidad (HRAE), siendo los lugares elegidos: Tuxtla Gutiérrez, Chiapas; Ciudad Victoria,
Tamaulipas; Mérida, Yucatán; Oaxaca, Oaxaca; Silao, Guanajuato, e Ixtapaluca, Estado de México. De ellos el único que no cuenta con Servicio pediátrico es el de Oaxaca, se desconoce el porqué, ya que dicho Estado tiene una población de más de 4 millones de habitantes, elevada natalidad y marginalidad con serios problemas socioeconómicos y culturales que hacen muy difícil el traslado de los pacientes cardiópatas a la Ciudad de México para su atención. Se puede decir en relación con la cirugía de CC en los diferentes HRAE lo siguiente: el hospital de Ixtapaluca fue inaugurado el 29 de marzo del 2012, a pesar de lo cual todavía hoy el volumen de pacientes pediátricos llevados a cirugía cardíaca es reducido, hay que recordar que el Estado de México es el más poblado del país y tiene según el INEGI 16 millones de habitantes, lo que obligaría con base en lo sugerido por la EACTS a que en dicha entidad hubiera cuatro centros capaces de llevar este tipo de intervenciones en un número de alrededor de 250 cirugías por año; el Hospital Regional de Alta Especialidad del Bajío cumple 11 años de existencia y si bien cuenta con los recursos tecnológicos y humanos necesarios, se desconoce el número de cirugías correctivas y paliativas de CC llevadas a cabo, el grado de complejidad y los resultados en términos de morbimortalidad, ya que no ha participado en el Registro Nacional de Cirugía Cardíaca Pediátrica (RENACCA$P E)$, ni en ninguna otra plataforma de información nacional o internacional. Otra situación enfrenta el HRAE de Tuxtla Gutiérrez, Chiapas, en el cual por variados problemas administrativos y sindicales el número de cirugías que se llevan a cabo es mínimo, lo que se ha subsanado, parcialmente, con el abordaje mediante cateterismo intervencionista (cierre de conducto arterioso y de comunicación interatrial, plastia de coartación aórtica y dilatación con balón en estenosis pulmonar, entre otras) y Jornadas Quirúrgicas llevadas a cabo por personal médico y paramédico de algún centro hospitalario de EE.UU., a este respecto es de mencionar que el Dr. Aldo Castañeda, un icono en la cirugía cardiovascular pediátrica mundial, desaprobaba lo que llamaba "safari médico", en el cual un grupo médicos y paramédicos de países desarrollados acuden a diversas regiones subdesarrolladas para llevar a cabo cirugía cardíaca en niños sin dejar un conocimiento sustantivo en los equipos de trabajo locales; el turismo quirúrgico debería limitarse a aquellos países o regiones de países que difícilmente tendrán la capacidad de contar con programas cardiotorácicos autónomos $^{22}$. En relación al HRAE de Ciudad Victoria, Tamaulipas, recientemente se agregó al grupo de trabajo 
un cirujano cardiovascular con subespecialidad en CC $y$ es de esperarse que paulatinamente se vaya logrando un mayor volumen quirúrgico. Finalmente, en cuanto al HRAE con sede en Mérida, Yucatán, los grupos médico y paramédico han empezado a tener ya resultados loables, no sin dificultades, en cuanto a un mayor número de cirugías, cateterismos intervencionistas y complejidad de los casos atendidos.

En 2007 quedó constituida la World Society for Pediatric and Congenital Heart Surgery (WSPCHS), con la misión de optimizar la atención de los pacientes con $\mathrm{CC}$ en todo el mundo. Para alcanzar dicho objetivo se planteó, como una de las estrategias, la creación de registros para conocer la situación mundial en relación con el tratamiento quirúrgico de los pacientes con CC. En México, en 2006, se creó la Asociación Mexicana de Especialistas en Cardiopatías Congénitas (AMECC), que congrega a la mayoría de los especialistas que se interesan y manejan las CC en nuestro país, como una asociación hermana de la WSPCHS. En la cumbre mundial de la WSPCHS celebrada en Montreal en el año de 2008 se hizo el compromiso de mejorar la atención cardíaca de los niños con CC en el mundo y la AMECC suscribió, con la representación del Dr. Samuel Ramírez Marroquín, ese enorme reto. Es relevante mencionar que en el artículo 5 de los estatutos de la AMECC se establece que su misión es la de organizar el trabajo colectivo con la participación de todas las instituciones gubernamentales y privadas cuya meta es el que todos los enfermos con un defecto cardíaco congénito que residan en el territorio nacional tengan acceso a una adecuada atención médica y quirúrgica. El Dr. Christo Tchervenkov, ex presidente de la WSPCHS resumió su visión del abordaje de la atención de las CC y la llamó la «Medicina del respeto», cuyos pilares son: responsabilidad, educación, sostenibilidad, asociación, empoderamiento, compromiso, trabajo en equipo y confianza (acrónimo RESPECT por sus siglas en inglés) ${ }^{24}$.

Con estos antecedentes, en el año 2010 se conformó un grupo colegiado de Cardiología y Cirugía Cardíaca Pediátrica dependiente de la Comisión Coordinadora de los Institutos Nacionales de Salud y Hospitales de Alta Especialidad (CCINSAHE) coordinado por el Dr. Manuel de la Llata en el que se establecieron varias acciones en el campo de las CC: la primera fue llevar a cabo el proyecto de regionalización en la atención quirúrgica de los niños aquejados con CC; la segunda acción fue realizar un censo de recursos humanos y tecnológicos para la atención de estos pacientes en el país. El censo mostró que el tratamiento de las CC en
México era en un $67 \%$ por cirugía y el resto por cateterismo intervencionista, y que un $85 \%$ de estos procedimientos se llevaban a cabo en los grandes centros hospitalarios de la Ciudad de México, Monterrey y Guadalajara. El total de cirugías cardiovasculares realizadas en el año 2009 fue de 4,000, lo que da un promedio de 38 cirugías por millón de habitantes, mientras que el promedio de cirugía de CC por cada millón de habitantes en Europa es de 62 y el de España de $52^{25}$. La tercera acción fue la creación de una base de datos, el RENACCAPE, que utilizando la nomenclatura internacional permitiera obtener la información del trabajo realizado en los diversos centros del país y permitiera cuantificar los avances logrados. Esta base de datos fue ideada y culminada con recursos propios por el Dr. Jorge Cervantes Salazar, y fue puesta a disposición, de manera gratuita, de la CCINSAHE y la AMECC $^{26}$. Con la información de la base de datos se logró la publicación del primer reporte de cirugía cardíaca pediátrica, que abarcó del 1 de agosto del 2011 al 31 de julio del 2012 y en el que se incluyó la información de prácticamente mil pacientes con la participación de las siguientes instituciones con los porcentajes respectivos: Instituto Nacional de Cardiología Ignacio Chávez (40\%); Hospital Infantil de México Federico Gómez (25\%); Instituto Nacional de Pediatría (16\%); Hospital Hidalgo en Aguascalientes(12\%); Hospital Regional de Alta Especialidad, Tuxtla Gutiérrez, Chiapas (6\%); Hospital Regional de Alta Especialidad en Mérida, Yucatán y Hospital Regional de Alta Especialidad de Oaxaca (0.4\%). Con respecto a los pacientes atendidos, el $10 \%$ correspondió a recién nacidos, el $29 \%$ a menores de 1 año y el $30 \%$ a niños de 1 a 5 años. La indicación quirúrgica fue electiva en el $89 \%$ y la mortalidad de la totalidad del grupo fue del $8 \% 25$.

En una publicación posterior se recapituló sobre los avances y pendientes en este proceso de regionalización en la revista de la WSPCHS. Dicha publicación mereció una editorial de la cardióloga pediatra Sara K. Pasquali, del Michigan Medicine Congenital Heart Center, y de Jeffrey P. Jacobs, Jefe de la División de Cirugía Cardiovascular del Johns Hopkins All Children's Heart Institute, en la que expresaron: «Se ha demostrado que la recopilación rigurosa de datos significativos para informar las mejores prácticas es un primer paso crítico, y la Asociación Mexicana de Especialistas en Enfermedades Congénitas del Corazón debe ser elogiada por dar este paso importante para mejorar la calidad de la atención en niños con cardiopatía congénita en México. Los futuros esfuerzos de colaboración entre RENACCAPE (base de datos mexicana) y la base 
de datos de cirugía cardíaca congénita de la Society of Thoracic Surgeons pueden facilitar aún más este objetivo. Esta base de datos ampliada podría mejorar y respaldar aún más el análisis de resultados y la mejora de la calidad en pacientes con enfermedad cardíaca congénita y pediátrica en América del Norte»27,28.

En la IV sesión científica de la WSPCHS, que tuvo lugar en Sao Paulo, Brasil, en 2014, se debatió sobre la necesidad de establecer una base de datos mundial cuyo objetivo sería el promover el conocimiento sobre la práctica global y los resultados de la cirugía cardíaca pediátrica y congénita; el contar con una herramienta para fomentar la mejora de la calidad en los hospitales participantes que no tuviera limitaciones geográficas 0 económicas con respecto a la elegibilidad para la participación y que fuera una fuente de información para evaluar el progreso en proveer la atención quirúrgica en niños con CC en el mundo. Este proyecto, World Data Base for Pediatric and Congenital Heart Surgery, cristalizó en 2017 y según el último reporte hay 48 centros hospitalarios inscritos que han informado sobre 3,436 cirugías, . En México participan a través del Instituto Nacional de Pediatría y del Centro Pediátrico del Corazón ABC-Kardias, del Programa Quirúrgico de Cardiopatías Pediátricas y del Instituto Nacional de Cardiología Ignacio Chávez ${ }^{29}$.

Existe otra base de datos, la International Quality Improvement Collaborative for Congenital Heart Disease (IQIC), que inició oficialmente en 2008 y cuyo fin era obtener información sobre la cirugía cardíaca congénita en entornos de recursos limitados, con el objetivo general de guiar los esfuerzos de mejoría de la calidad y reducir la mortalidad quirúrgica de las $\mathrm{CC}$. Hoy en día, hay 64 centros inscritos de 25 países en el IQIC. México participa a través del Instituto Nacional de Pediatría y del Programa Quirúrgico de Cardiopatías Pediátricas de Kardias A.C. y el Centro Médico $A_{B C}{ }^{30}$.

El grupo colegiado de lo que se llamó Consejo Coordinador de la Regionalización de la Atención de Cardiopatías Congénitas permaneció en funciones hasta el año de 2017, después de haber llevado a cabo, aparte de lo ya mencionado, dos reuniones nacionales con foros de análisis y discusión sobre temas de gran importancia para el campos de la cardiología y cirugía cardiovascular pediátricas. Desafortunadamente el grupo se disolvió sin haber logrado los objetivos para los que fue formado, ya que las autoridades consideraron otras prioridades en políticas de salud.

La atención quirúrgica de las CC en nuestro país se ha ido subsanando en diversas formas por el esfuerzo de Médicos y Sociedades Civiles. Es de destacar lo realizado en el Centenario Hospital Miguel Hidalgo en Aguascalientes (12\%), donde se está llevando a cabo un programa de cirugía cardíaca pediátrica con un buen volumen de casos operados y, cada vez, con un mayor grado de complejidad. En esta entidad se ha aplicado una herramienta útil: el «programa de acompañamiento", con el Instituto Nacional de Cardiología Ignacio Chávez, que consiste en analizar los casos de mayor complejidad y previo consenso decidir sobre el abordaje quirúrgico y la institución donde se llevará a cabo el procedimiento. En caso de ser requerido, alguno de los cirujanos cardiovasculares del Instituto Nacional de Cardiología Ignacio Chávez así como cirujanos y anestesiólogos participan con el equipo quirúrgico local en Aguascalientes. También se están realizando cateterismos intervencionistas con el valioso apoyo del Dr. José Antonio García Montes. Este programa es resultado del empuje y el entusiasmo de los cardiólogos pediatras, en especial de la Dra. Julieta González Palacios, y cirujanos cardiovasculares de dicha institución y los recursos financieros que sustentan el programa son estatales y del Seguro Popular. Este programa ha permitido que se manejen la gran mayoría de las CC del Estado y que tenga una positiva influencia en estados circunvecinos como el de Zacatecas. Un esquema similar se lleva a cabo en otros nosocomios como el Hospital Pediátrico de Sinaloa, que recibe apoyo de cirujanos cardiovasculares de Monterrey o de la Ciudad de México cuando así es necesario.

Otra experiencia a mencionar es la del Hospital General de Mexicali en Baja California, en donde se ha logrado un programa exitoso de cirugía cardíaca pediátrica, mediante jornadas quirúrgicas, en las que participa un equipo especializado de cirujanos cardiovasculares del Instituto Nacional de Cardiología Ignacio Chávez, y cirujanos y anestesiólogos cardiovasculares, hemodinamistas e intensivistas locales. Han alcanzado un excelente volumen de cirugías cardíacas que ha incluido cirugía cardíaca neonatal. Este programa se puede denominar como híbrido por el origen de los recursos financieros provenientes del Seguro Popular, de recursos estatales y la Fundación Médicos con Corazón, siendo eficientemente administrados por el Patronato del Hospital General de Mexicali. Merece una mención especial la enorme labor administrativa y de coordinación desarrollada por el Dr. Hugo Martínez Espinoza.

Otro programa híbrido desarrollado en la Ciudad de México es el promovido por la fundación Kardias y el Centro Médico ABC, Institución de Asistencia Privada (IAP) y encabezado por el cirujano cardiovascular Alexis 
Palacios Macedo. Los recursos financieros son suministrados por la Fundación, el Hospital y el Seguro Popular. Es de mencionar que dicho programa se lleva a cabo todo el año y cuenta con el apoyo estratégico del Hospital de Niños de Texas. De ser un programa netamente quirúrgico, desde el segundo semestre del 2017 ha sido complementado con cateterismo intervencionista. Otro programa con recursos privados en su totalidad es el llamado Corazones Invencibles, llevado a cabo mediante jornadas quirúrgicas o de cateterismo intervencionista en el Hospital Zambrano Hellion en Monterrey, con el apoyo económico de la fundación TecSalud y la Asociación CardioChavitos, con la participación voluntaria de médicos especialistas de la localidad, profesionales de la salud de los hospitales San José y Zambrano Hellion y médicos de la International Children's Heart Foundation. Así mismo, en el $\mathrm{H}+$ de los Cabos, la Fundación Corazón de Niño realiza Jornadas denominadas Unidos de Corazón de cateterismos intervencionistas, estudios electrofisiológicos terapéuticos y cirugías cardíacas pediátricas con recursos donados por la sociedad civil y con el apoyo de dicho hospital. En estas jornadas participan médicos especialistas de diversos estados de la República y, en forma paralela, se realizan jornadas de diagnóstico en CC.

Es necesario hacer alguna consideración sobre los programas de «hermandad» en los cuales se establece un acompañamiento de dos programas cardíacos, uno un centro de excelencia establecido y el otro algún programa en evolución en un país en desarrollo para establecer una relación de valor para ambas instituciones. En aquellos países donde uno de los problemas principales es el deficiente acceso a la atención, los centros de excelencia existentes pueden contribuir a la creación y el apoyo de nuevos centros, lo que se podría denominar programas nacionales de hermandad o de tutoría. Los programas de acompañamiento internacional son primordiales en países sin centros capaces de proporcionar ese apoyo. Los siguientes puntos son esenciales para alcanzar éxito en dicha asociación: a) tener el objetivo de trabajar en equipo y que incluya compartir la responsabilidad y establecer un intercambio abierto de información, los esfuerzos deben enfocarse en la educación y sostenibilidad del programa; b) establecer la necesidad de medir los resultados de sus servicios cardiotorácicos para mejorar el programa, para lo cual se debe contar con una base de datos fiable cuyos resultados deberán ser revisados de manera periódica por la institución con las metas claras de aumentar el volumen quirúrgico, mejorar la calidad y reducir la morbilidad y la mortalidad; c) idealmente contar con una estructura de recaudación de fondos, llámese organizaciones de la sociedad civil o fundaciones locales con un manejo trasparente de estos que permita su adecuada canalización y utilización de los recursos, y d) construir puentes para obtener el apoyo gubernamental y comunitario local para la sostenibilidad del programa con objetivos establecidos a mediano y largo plazo ${ }^{31}$.

Existen otras asociaciones orientadas a la atención de niños de escasos recursos que padecen una afección cardíaca y que requieren de una cirugía cardíaca correctiva o cateterismo intervencionista, como la Fundación Derechos de la Infancia creada por el Ing. Luis Martínez Zepeda (1915-2010), quien se formó en el Instituto Politécnico Nacional y tenía una visión social muy profunda. Esta fundación ha apoyado de manera decidida y con importantes recursos la atención de niños cardiópatas en diversos hospitales: Instituto Nacional de Cardiología, Hospital Infantil de México, Instituto Nacional de Pediatría y el Hospital de Especialidades Pediátricas de Tuxtla Gutiérrez. La Asociación de Ayuda de Niños en Estado Crítico (ADANEC), con más de dos décadas de existencia, ha hecho una labor loable en la atención quirúrgica de los niños aquejados con algún tipo de CC. Esta asociación lleva a cabo jornadas quirúrgicas en diversas partes del país y las de mayor complejidad se realizan en el Hospital de Alta Especialidad Materno Infantil en Monterrey por el equipo de salud de ADANEC. En este recuento es importante mencionar a la Fundación Gonzalo Río Arronte, la cual tiene un amplio reconocimiento en el Sector de la Salud por su contribución en la modernización tecnológica y la ampliación de la cobertura de los servicios médicos en nuestro país. Proporciona donativos en especie a instituciones, hospitales, clínicas y centros de salud que prestan servicios de salud a población de escasos recursos o en situación de vulnerabilidad.

Con los ejemplos arriba señalados es evidente que la sociedad civil ha apoyado en forma decidida la atención de los niños con patología cardíaca congénita, dicha labor ha sido de gran relevancia, pero el objetivo de atender a todos los niños con CC de manera oportuna y con calidad no se podrá alcanzar a pesar de los valiosos afanes de todas estas organizaciones que trabajan en forma admirable pero de manera aislada. Como bien lo expresan Dearani, et al..$^{32}$, el propósito de mejorar el acceso a la atención de los niños con CC solo se podrá lograr mediante la construcción de alianzas de grupos y con una coordinación de sus esfuerzos. Es necesario promover la cooperación, no la competencia, ya que esta provoca duplicaciones innecesarias de 


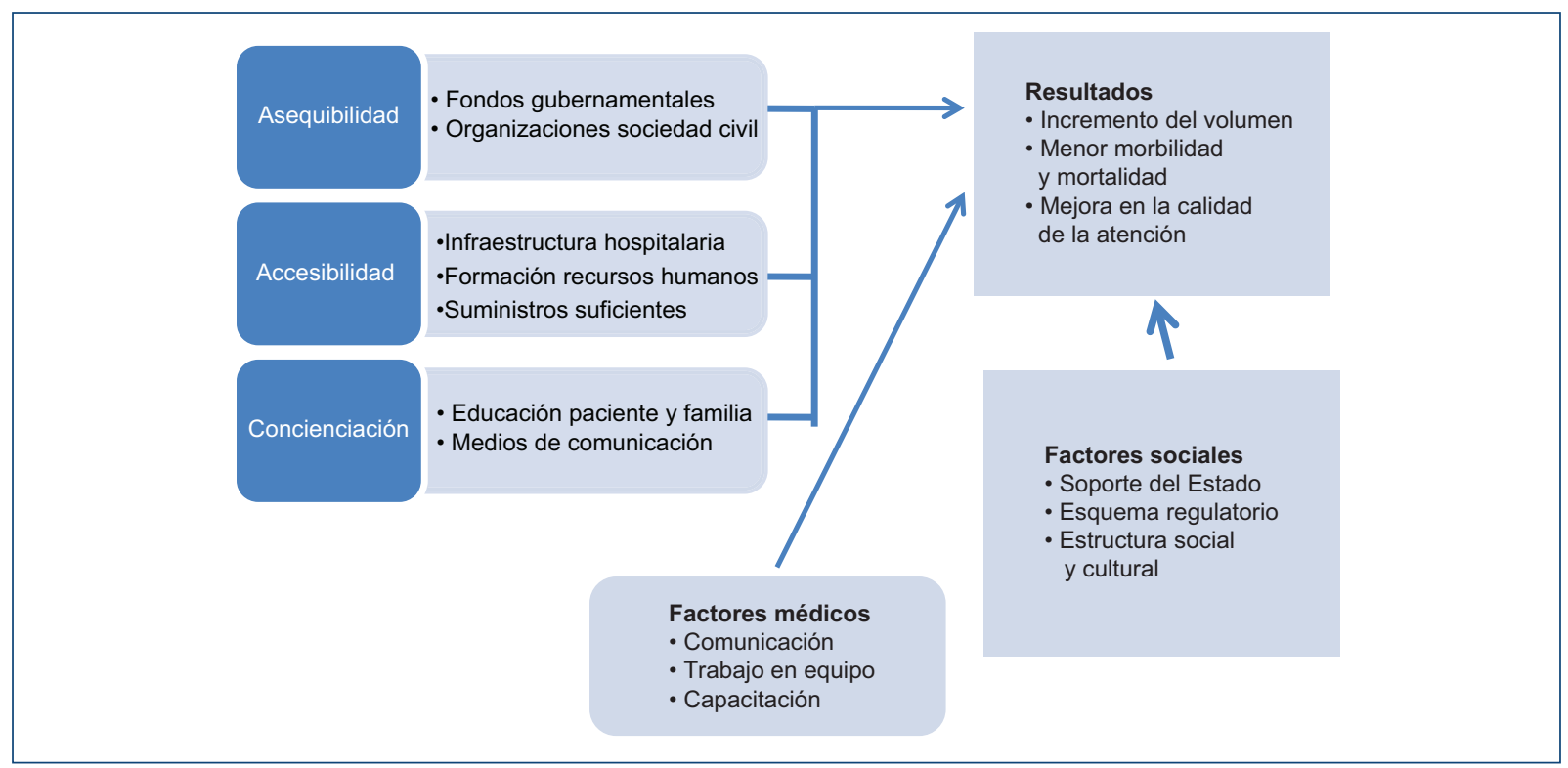

Figura 4. Prioridades en la atención de las cardiopatías congénitas (adaptada de Dearani, et al., 2016).31

esfuerzos y termina siendo ineficiente. Sería deseable establecer puentes entre las diferentes organizaciones civiles e instituciones de salud para intercambiar ideas que permitan crear consensos y proyectos conjuntos para una mejor utilización de los recursos disponibles.

Como colofón se puede concluir que en el ámbito de la política de salud, la Secretaría de Salud debe considerar la atención de las CC como una prioridad nacional con el fin de que se le destinen mayores recursos financieros, con un manejo administrativo muy eficiente para construir infraestructura hospitalaria suficiente así como fortalecer y consolidar, de manera estratégica, a los diferentes grupos de trabajo de hospitales regionales ya existentes, debiendo incrementar el apoyo a los Institutos Nacionales de Salud para que continúen como centros generadores de conocimiento y sedes para la formación de recursos humanos especializados y centros que acompañen a diversos programas de cirugía cardíaca pediátrica y cateterismo intervencionista en el país. Se debe lograr la accesibilidad, la disponibilidad y la asequibilidad de la atención quirúrgica 0 de cateterismo intervencionista de las $\mathrm{CC}^{33}$. Las metas en la esfera asistencial son: a) lograr la atención de los niños aquejados con CC en todo el país; b) consolidar equipos de trabajo en los Hospitales Regionales de Alta Especialidad (HRAE); c) fortalecer Hospitales Estatales con capacidad para llevar a cabo cirugía cardíaca pediátrica; d) utilizar la base de datos nacional RENACCAPE para contar con una herramienta que permita conocer la labor desarrollada en este campo en el país y que permita evaluar los avances, y e) obtener resultados adecuados comparados con los estándares internacionales. Desde el punto de vista social se debe orientar la mirada de la sociedad sobre la importancia de las CC en nuestro país (Fig. 4).

Es necesario reflexionar sobre los retos que debemos afrontar y recordar lo que Sir George Alleyne, Premio Ciencia de la Paz de la Fundación Interamericana del Corazón (2008) y Doctor Honoris Causa por la Universidad de Toronto (2017), atinadamente afirmó6: «La equidad en materia de salud es fundamentalmente un tema ético-moral y no debemos negarnos a considerarlo así».

\section{Financiación}

La presente investigación no ha recibido ayudas específicas provenientes de agencias del sector público, sector comercial o entidades sin ánimo de lucro.

\section{Conflicto de intereses}

El autor hacer constar que no posee ningún conflicto de intereses.

\section{Responsabilidades éticas}

Protección de personas y animales. El autor declara que para esta investigación no se han realizado experimentos en seres humanos ni en animales. 
Confidencialidad de los datos. El autor declara que en este artículo no aparecen datos de pacientes.

Derecho a la privacidad y consentimiento informado. El autor declara que en este artículo no aparecen datos de pacientes.

\section{Bibliografía}

1. Knaul FM, Arreola-Ornelas H, Méndez-Carniado O. Protección financiera en salud: actualizaciones para México a 2014. Salud Pública Mex. 2016:58:341-50.

2. Health spending. Related topics [Internet]. Organisation for Economic Co-operation and Development; 2018. Disponible en: https://data.oecd. org/healthres/health-spending.htm.

3. Global Health Expenditure Database. World Health Organization Expenditure database [Internet]. Ginebra: World Health Organization [fecha de última actualización: 4 de abril de 2019]. Disponible en: http://apps.who. int/nha/database.

4. Frenk J, Knaul FM, Gómez Dantes O, Arreola Ornelas H. La brecha de la salud. Nexos. 2018;40(482):20-5.

5. Cano Valle F, Pantoja Nieves M, Sánchez Miguel M. El Péndulo de Medicina. El doble péndulo de la salud. Urge una reforma en el sistema de salud. México: Editorial Prado, Instituto de Investigaciones Jurídicas; 2017.

6. Cano Valle F, coord. La Salud universal... una entelequia. La posición de la Academia ante el Sistema NAcional de Salud. México: Academia Nacional de Medicina; 2018. pp. 15-28.

7. Organization for Economic Co-operation and Development. (OECD) (Internet). Reviews of Health Care Systems: Mexico 2016. Disponible en: https://www.oecd.org/health/health-systems/OECD-Reviews-ofHealth-Systems-Mexico-2016-Assessment-and-recommendations-Spanish.pdf.

8. Cutler D. Buried in healthcare imbroglio, trillion-dolar question [Internet]. The Harvard Gazzete; 2017. Disponible en: https://news.harvard.edu/ gazette/story/2017/08/looking-beyond-d-c-debate-harvard-economist-zeroes-in-on-cost/

9. Bloom DE, Canning D, Sevilla J. The effect of health on economic growth: a production function approach. World Development. 2004;32(1):1-13.

10. Frenk J, González-Pier E, Gómez-Dantes O, Lezana MA, Knaul FM. Comprehensive reform to improve health system performance in Mexico. Lancet. 2006;368:1524-134.

11. Ávila-Burgos L, Serván-Mori E, Wirtz VJ, Sosa-Rubí S, Salinas-Rodríguez A. Efectos del Seguro Popular sobre el gasto en salud en hogares mexicanos a diez años de su implementación. Salud Publica Mex. 2013; 55(suppl 2):S91-9.

12. Knaul FM, Arreola-Ornelas H, Méndez-Carniado O, Bryson-Cahn C, Barofsky J, Maguire R, et al. Evidence is good for your health system: policy reform to remedy catastrophic and impoverishing. Lancet. 2006;368:1828-41.

13. Organization for Economic Co-operation and Development (OECD) (Internet). MEXICO Country Highlights OECD, Doing Better for Children. Disponible en: https://www.oecd.org/mexico/43590178.pdf

14. Información obtenida de Indicadores Mundiales - IndexMundi. Disponible en: https://www.indexmundi.com/g/g.aspx?c=mx\&v=29\&l=es

15. Calderón-Colmenero J, Ramírez-Marroquín S, Cervantes-Salazar J. Métodos de estratificación de riesgo en la cirugía de cardiopatías congénitas. Arch Cardiol Méx. 2008;78:60-7.
16. O'Brien SM, Clarke DR, Jacobs JP, Jacobs ML, Lacour-Gayet FG, Pizarro C et al. An empirically based tool for analyzing mortality associated with congenital heart surgery. J Thorac Cardiovasc Surg. 2009;138(5):1139-53.

17. Calderón-Colmenero J, Cervantes-Salazar JL, Curi-Curi JP, Ramírez-Marroquín S. Problemática de las cardiopatías congénitas en México. Propuesta de regionalización. Arch Cardiol Mex. 2010;80(2):133-40.

18. Mitchell SC, Korones SB, Berrendees HW. Congenital heart disease in 56,109 births. Incident and natural history. Circulation. 1971;43:323-32.

19. Fyler DC. Report of the New England regional infant cardiac program. Pediatrics. 1980;65(suppl):S376-S61.

20. Hoffman JIE, Kaplan S. The incidence of congenital heart disease. J Am Coll Cardiol. 2002;39:1890-9.

21. Torres-Cosme JL, Rolón-Porras C, Aguinaga-Ríos M, Acosta-Granados PM, Reyes-Muñoz E, Murguía-Peniche T. Mortality from congenital heart disease in Mexico: A problem on the rise. PLoS One. 2016:11(3):e0150422.

22. Sánchez-Barriga JJ. Mortality trends from congenital malformations of the heart and great vessels in children and adults in the seven socioeconomic regions of México, 2000-2015. Congenit Heart Dis. 2018; 13(5):690-9.

23. Daenen W, Lacourt-Gayet F, Aberg T. Optimal structure of a congenital heart surgery department in Europe by EACTS congenital heart disease committee. Eur J Cardiothorac Surg. 2003;24:334-51.

24. Jacobs JP, Tchervenkov CI, Stellini G, Kurosawa H, Mavroudis C, Jatene MB, et al. Historial of the World Society for Pediatric and Congenital Heart surgery: the first decade. World J Pediatr Congenit Heart Surgery. 2018;9(4):392-06.

25. Calderón-Colmenero J, De la Llata M, Vizcaíno A, Ramírez S. Atención médico-quirúrgica de las cardiopatías congénitas: una visión panorámica de la realidad en México. Encuesta 2009. Rev Invest Clin. 2011;63(4): 344-52.

26. Cervantes-Salazar J, Calderón-Colmenero J, Ramírez-Marroquín, Palacios-Macedo A, Bolio-Cerdán A, Vizcaíno A, et al. Registro Mexicano de Cirugía Cardiaca Pediátrica. Primer Informe. Rev Invest Clin. 2013;65(6): 476-82.

27. Calderón-Colmenero $J$, Cervantes-Salazar $J$, Curi-Curi $P, \quad R a-$ mírez-Marroquín S. Congenital heart disease in Mexico: Advances of the Regionalization Project. World J Pediatr Congenit Heart Surg. 2013; 4(2):165-71.

28. Pasquali SK, Jacobs JO. The role of databases in improving the quality of care for congenital heart disease. World J Pediatr Congenit Heart Surg. 2013;4(2):139-41.

29. St. Louis JD, Kurosawa $H$, Jonas RA, Sandoval N, Cervantes J, Tchervenkov $\mathrm{Cl}$, et al. The World database for pediatric heart surgery. The Dawn of a new era of global communication and quality improvement in congenital heart disease. World J Pediatr Congenit Heart Surgery. 2017; 8(5):597-99.

30. International Quality Improvement Collaborative [Internet]. Minneapolis, MN: Children's HeartLink; 2018. Disponible en: https://childrensheartlink. org/iqic/

31. Dearani JA, Jacobs JP, Bolman M, Swain JD, Vricella LA, Weinstein S, et al. Humanitarian outreach in cardiothoracic surgery: From setup to sustainability. Ann Thorac Surg. 2016;102:1004-11.

32. Dearani JA, Neirotti R, Kohnke EJ, Sinha KK, Cabalka AK, Barnes RD, et al. Improving pediatric cardiac surgical care in developing countries: Matching resources to needs. Semin Thorac Cardiovasc Surg Pediatr Card Surg Ann. 2010;13:35-43.

33. Saxton AT, Poenaru D, Ozgediz D, Ameh EA, Farner D, Smith ER, et al. Economic analysis of children's surgical care in low- and middle-income countries: A systematic review and analysis. PLoS ONE. 2016;11(10):1-20. 